九州大学学術情報リポジトリ

Kyushu University Institutional Repository

\title{
Future Prospect of Plumbene: A Review
}

\section{Megha Gupta Chaudhary}

Department of Physics, SRM Institute of Science and Technology, NCR Campus, Delhi-NCR Campus

Kumar, Nitin

Department of Electronics and Communication Engineering, SRM Institute of Science and Technology, NCR Campus, Delhi-NCR Campus

\section{Kumar, Sushil}

Department of Physics, SRM Institute of Science and Technology, NCR Campus, Delhi-NCR Campus

https://doi.org/10.5109/4742116

出版情報：Evergreen. 8 (4)，pp.732-739，2021-12. 九州大学グリーンテクノロジー研究教育センター バージョン：

権利関係 : 


\title{
Future Prospect of Plumbene: A Review
}

\author{
Megha Gupta Chaudhary ${ }^{1}$, Nitin Kumar $^{2 *}$ and Sushil Kumar ${ }^{1}$ \\ ${ }^{1}$ Department of Physics, SRM Institute of Science and Technology, NCR Campus, Delhi-NCR Campus, Delhi- \\ Meerut Road, Modinagar, Ghaziabad, UP, India \\ ${ }^{2}$ Department of Electronics and Communication Engineering, SRM Institute of Science and Technology, NCR \\ Campus, Delhi-NCR Campus, Delhi-Meerut Road, Modinagar, Ghaziabad, UP, India
}

*Author to whom correspondence should be addressed:

E-mail: nitindhama85@gmail.com

(Received June 5, 2021; Revised September 27, 2021; accepted October 24, 2021).

\begin{abstract}
Discovery of graphene and its bewildering properties have brought forth another class of materials known as "two dimensional materials" or 2D materials. Spurred by the achievement of graphene, alternative layered and non-layered two dimensional materials have become the focal point of extraordinary research because of their novel physical and chemical properties. Plumbene, a single layer of lead $(\mathrm{Pb})$ is the latest two dimensional material, has pulled in huge enthusiasm inferable from its uncommon properties and possible applications. In this paper, we investigation about the most recent advancement in the investigation of Plumbene, for the most part on the structure, growth techniques, physical properties, future applications and difficulties. The phenomenal mechanical and thermal properties of Plumbene will open the best approach to batteries, machine manufacturing, shipbuilding, etc. Lastly, this review concludes with a future forthcoming to manage this quick advancing class of two dimensional materials in cutting edge materials science.
\end{abstract}

Keywords: Two-Dimensional layered materials; Plumbene; Topological Insulators; Spintronics

\section{Introduction}

The commendable achievement of graphene ${ }^{1-4)}$, that's few of the firsts of these two-dimensional (2D) materials has started a runway impact in the quest for examining other 2D materials ${ }^{5}$. After the discovery of graphene, numerous researchers would not realize that a dozen 2D materials such as silicene $e^{6-8)}$, germanene ${ }^{9-11)}$, and phosphorene $^{12-16)}$ and stanene ${ }^{17-21)}$ etc. can be isolated and studied in the past two decades. The latest 2D material in this series is Group 14 element i.e. Plumbene ${ }^{22)}$, a monolayer of lead, having honeycomb structure is added. The chemical symbol of Lead is $\mathrm{Pb}$, originating from the Latin word "plumbum" for lead; in this manner, a monolayer of the $\mathrm{Pb}$ film can be called "plumbene," in similarity with graphene and silicene. As the primary individual from the 2D family; the utilization of graphene has advancement over an expanded division of uses going from unconventional electronics gadgets $^{23)}$ to biomedicine ${ }^{24-27)}$ because of its exceptional properties, for example, ultra-high carrier mobility ${ }^{28)}$, ultra-low weight ${ }^{29)}$, high thermal conductivity ${ }^{30)}$, high optical transparency ${ }^{31)}$, flexibility $^{32)}$ and remarkable high strength ${ }^{33)}$. Regardless, graphene has tunable intriguing electronic properties ${ }^{34)}$, the spin-orbit (SO) ${ }^{35-36)}$ coupling is feeble restricting its uses as spin filters ${ }^{37}$, topological insulators (TIs) ${ }^{38)}$ and so forth. In this way, there is a critical demand to find novel
2D materials in after graphene to beat its lacks.

Topological insulators ${ }^{39-40)}$ due to their nature, enforce the electrons to move on the surface at very high speeds subsequently concluding valuable uses in electronics and photonic devices. Investigation of the group 14 elements ${ }^{41)}$, utilizing first-principles computations has uncovered enormous interest because it has the highest spin-orbit interaction, because of orbital electron structure of lead and consequently the highest energy band gap, which makes it a robust 2D topological insulator wherein the Quantum Spin Hall Effect ${ }^{42)}$ may arise even above room temperature.

\section{Crystal Structure}

The information about the stability of crystal structure and surface properties are basis for any 2D material if it is to have feasible utilization in any area of interest. This segment presents an understanding of the fundamental crystal arrangement of elemental structures.

In the different investigations related to the structure of Group IV components (C, Si, Ge, Sn and $\mathrm{Pb})^{43}$ ), it was proposed that the atomic arrangement of planar Group IV elements follow the hexagonal honeycomb structure of graphene ${ }^{44-45)}$.Plumbene moreover has buckled honeycomb structure similar to graphene and silicene. Figure. 1a outlines the hexagonal structure and the 
buckled nature of plumbene. The lattice constant of plumbene is recorded as $4.93 \AA^{22)}$, and buckling height $(\delta)$ is estimated between $0.97 \AA$ to $1.01 \AA^{46)}$. The $\mathrm{Pb}-\mathrm{Pb}$ bond distance (d) i.e the nearest neighbour atoms in plumbene is $3.00 \AA^{22)}$, calculated by utilizing structural optimization. The bond angle between $\mathrm{Pb}-\mathrm{Pb}$ atoms in plumbene is $108.34^{\circ 22}$ which outcomes in buckled structure of plumbene. Overlapping between $\pi-\pi$ bonds are additionally determined in plumbene analogous to graphene however weaker in nature than graphene due to longer bond length. In plumbene, due to its buckled configuration, overlapping between $\pi$ and $\sigma$ orbitals is higher, and accordingly the system is stabilized ${ }^{46)}$. A high band gap $(\sim 0.4 \mathrm{eV})^{47)}$ which is lots large than that of other group-IV 2D materials is detected in plumbene and subsequently can be a perfect material for a topological insulator.

It can furthermore have uses related to machine manufacturing, shipbuilding, and in batteries etc., wherein convectional lead is utilized so far. large band gaps are anticipated in decorated plumbene48), which might lead to a quantum spin hall insulator ${ }^{49)}$ behaviour and captivating high-temperature applications. Certainly, synthesizing such sheets whereas maintaining their nontrivial properties remains a difficult task.

a)

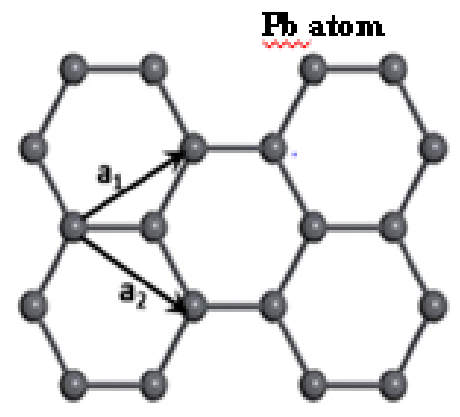

b]

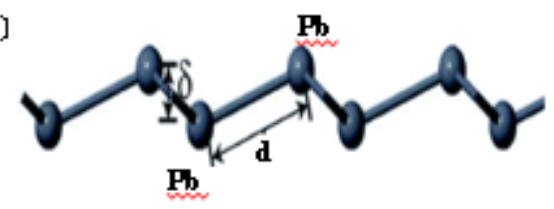

Figure. 1:(a) Top and (b) side view of honeycomb structure of plumbene, where $\mathrm{a}_{1}$ and $\mathrm{a}_{2}$ are the primitive vectors, $\delta$ is buckling height and $\mathrm{d}$ is the distance between $\mathrm{Pb}-\mathrm{Pb}$ bonds. a)

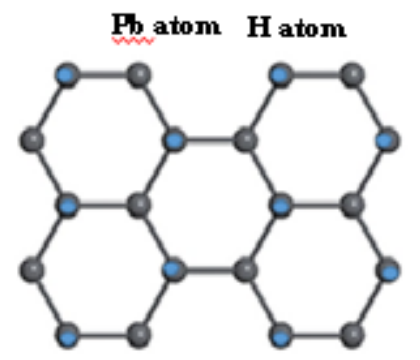

b)

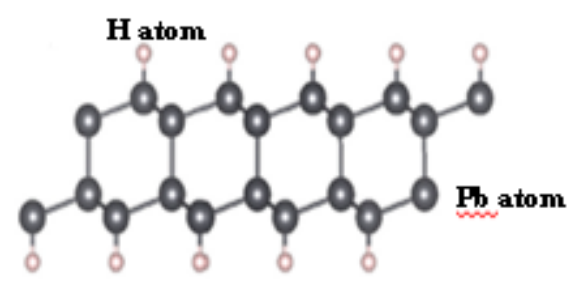

Figure. 2: (a) Top and (b) side view of the bilayer plumbene with $\mathrm{Pb}$ and $\mathrm{H}$ atoms, respectively.

The structure of different two dimensional group IVA materials with chemical functionalization have moreover been considered broadly, and a few with huge bulk gaps have been anticipated theoretically ${ }^{50-54)}$. Moving from graphene to plumbene, the buckling height is found to be extended and the increase in atomic mass also prompts to a considerable rise in the effective spin orbit coupling $(\mathrm{SOC})^{55)}$. It is notable that SOC assumes a significant part in quantum spin hall (QSH) insulators. Recently, A special class of 2D QSH insulators in X-decorated plumbene monolayers (PBX; X = H, F, Cl, Br, I) with unusually enormous bulk gaps from $1.03 \mathrm{eV}$ to most elevated estimation of $1.34 \mathrm{eV}$ has been predicted ${ }^{47)}$.Figure. 2 illustrates the structure of the bilayer plumbene, in which all adjacent $\mathrm{Pb}$ atoms are $\mathrm{sp}^{3}$ hybridized. Remarkably, the optimized buckled height $(\delta)$ is found $0.82 \AA^{49)}$ comparative to the case $\left.(0.78 \AA)^{47}\right)$ of monolayer plumbene. Also, a new family of methyl and trihelogenomethyl decorated plumbene monolayer ${ }^{48)}$ i.e. monolayer with $-\mathrm{CX} 3$ where, $\mathrm{X}=\mathrm{H}, \mathrm{F}, \mathrm{Cl}$ is investigated and report exceptionally huge band gaps within the range of $0.84 \mathrm{eV}$ to $0.98 \mathrm{eV}$ with spin-orbit coupling. These range of band gaps is considerable higher in comparison to many existing topological insulators and feasible at room temperature.

\section{Fabrication Process}

After the synthesis and characterization of graphene, there are various two dimensional materials which have been arduously examined with the objective of applying unique promising 2D material's properties ${ }^{3,7,8,19-20)}$. After the revolutionary growth of silicene on $\mathrm{Ag}(111)^{8)}$, the, down the column, group 14 mono elemental two dimensional honeycomb lattices were at the core interest. Before long, germanene and stanene, as well as silicene over again, have been acknowledged on different surfaces. 
In addition, group 13and 15 analogous such as borophene $^{56-58)}$, phosphorene ${ }^{59)}$, antimonene ${ }^{60)}$, and bismuthene $^{61)}$, have been synthesized too. The acknowledgement of the remaining material in the group 14 elements, i.e., plumbene, persisted a pursuit for the ambrosia, in spite of the fact that numerous hypothetical research forecast its stability and expected extraordinary intriguing properties.

In recent times, the synthesis of large area plumbene sheets were reported by Yuhara and co-workers ${ }^{62)}$. The synthesis has been carried out by the epitaxial growth of plumbene by way of segregation from prepared $\mathrm{Pd}_{1-\mathrm{x}} \mathrm{Pb}_{\mathrm{x}}$ (111) alloys grown on Pd (111) substrates upon heating/alloying $\mathrm{Pb}$ thin films deposited ${ }^{62)}$ at room temperature (Refer Figure. 3). The establishment and structure of plumbene sheets so acquired used to be examined via the usage of low-energy electron diffraction (LEED) and scanning tunnelling microscopy (STM) which approves that a $\mathrm{Pb}$ sheet overlays the $\mathrm{Pd}_{1 \text { - }}$ ${ }_{x} \mathrm{~Pb}_{\mathrm{x}}$ (111) alloy and exhibit a planar honeycomb structure with a unit cell in accordance to that of freestanding plumbene.

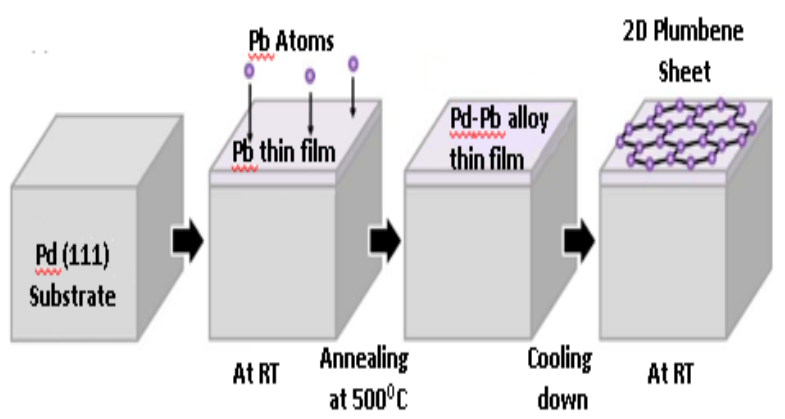

Figure. 3: Epitaxial growth of 2D honeycomb plumbene sheets ${ }^{62)}$.

\section{Properties of plumbene}

This segment presents the progressing status on the comprehension of physical, electronic and magnetic properties of plumbene. The band gap in plumbene is much higher than other existing $2 \mathrm{D}$ materials such as graphene and silicene ${ }^{47)}$. Consequently, they are able to have possible applications including topological insulators and high-temperature operations. Structural configurations moreover bring about beneficial irreplaceable properties of plumbene than other two dimensional materials. Indeed, surprisingly it has prevalent properties in comparison to lead which can be used for the utility in batteries, machine manufacturing, shipbuilding, and so on, wherein traditional lead is utilized so far. Plumbene can too utilized for power generations, preparation of plumbene-reinforced nanocomposites, etc. Plumbene will have anticipated uses wherein conductivity is the major issue. Plumbene's thermal and mechanical properties are additionally described recently.

\subsection{Electronic and topological properties}

Topological insulators (Tis) ${ }^{38-39,42)}$, also known as quantum spin hall (QSH) insulators ${ }^{39-40)}$, as a novel class of quantum materials ${ }^{55}$ have caused exhaustive investigation in quantum information, as they provide an unconventional and strong ground for attaining relativistic and spin-polarized Fermions in the condensed matter system.

As of now, two dimensional materials in groups IV and $\mathrm{V}$ are favourable applicant for the quantum spin hall effect characterized by using an insulating bulk-gap and gapless edge states at its boundaries due to time-reversal symmetry (TRS) ${ }^{55}$, hence giving the alluring ideas to unique quantum electronic devices with low energy dissipation. Earlier studies have proved that2D group IV elements, such as graphene ${ }^{63,64)}$, silicene ${ }^{65,66)}$, germanene ${ }^{67,}$ 68), and stanene ${ }^{69,70)}$ are topological insulators ${ }^{55)}$ but plumbene is considered as a normal band insulator due to excessive strong SOC effect ${ }^{22}$.

Theoretical calculations foresee that a giant bulk gap can be acquired via fluorination, hydrogenation, or different sorts of functionalizations ${ }^{47,48)}$. Absorption of atom or molecule is required to saturate the $p_{z}$ orbital or dangling bond so that a band gap can be released at the Dirac point except SOC. At the point, when the SOC is thought of, a larger gap can be acquired in the corresponding system. For instance, first-principles calculations forecast a 2D QSH insulator in F-decorated plumbene monolayer with an exceptionally large bulk gap of $1.34 \mathrm{eV}^{47-49)}$.

First-principles and tight-binding computation are used to explore plumbene's band structures and topologicalproperties ${ }^{22)}$. Results show that the system can change from a normal insulator to topological insulator with change in large bulk gap of $\sim 400 \mathrm{meV}$ to $\sim 200$ $\mathrm{meV}^{22}$. The QSH state is exceptionally strong with regard to external strain. Due to plumbene's simple structure, and external field effects on two large bulk gaps, it can be a favourable ground for topological phenomena and noval quantum device uses at room temperature.

Additionally, 2D materials with coexisting topological and ferroelectric ordering have been the centre of 2D condensed matter physics in late years, and their concurrence in a single system may make vital undiscovered development and potential uses. Recently, a class of ferroelectric topological insulators (FETIs) in $\mathrm{CH}_{2} \mathrm{OCH}_{3}$-functionalized plumbene $\left(\mathrm{Pb}-\mathrm{CH}_{2} \mathrm{OCH}_{3}\right)^{71)}$ is illustrated on the basis of first-principles calculations, which confirms the huge nontrivial band gap of $0.80 \mathrm{eV}$ in $\mathrm{Pb}-\mathrm{CH}_{2} \mathrm{OCH}_{3}$, made acquainted by the orbital filtering effect in the existence of spin-orbit coupling (SOC). These outcomes at the aggregate of topological and ferroelectric characteristics may give a new platform for the advancement of microelectronic devices and the use of spintronics at room temperature. Recently, plumbene on Fe layer has been explained theoretically. Fe layer being a ferromagnetic element prompts a noteworthy exchange 
splitting in plumbene due to which, there is a transition from quantum spin Hall to quantum anomalous Hall state $^{72)}$.

\subsection{Magnetic Properties}

It has been appeared that the $2 \mathrm{D}$ semiconductors combining with magnetic data storage materials might prompt to 2D spintronics devices with huge applications ${ }^{73)}$. The magnetic properties of 2D materials may be altered with the aid of using doping process or making adsorption of transition metal elements that could result in dilute magnetic semiconductors in 2D semiconductors ${ }^{74)}$.

To investigate the magnetism in plumbene, 3d transition metal elements such as Ti, V, Cr, Mn, Fe, and Co are doped in plumbene ${ }^{75)}$ which shows magnetic states in monolayer plumbene. All the computation have been accomplished the use of spin polarized density functional theory (DFT) including SOC, and Vienna ab initio simulation package (VASP). It was also observed that Sc and Ni-doped plumbene systems do not show magnetic solutions. The values of spin magnetic moments vary from $1.32 \mu \mathrm{B}$ (minimum) to $4.10 \mu \mathrm{B}$ (maximum) for different doping elements as mentioned above.

For practical spintronic applications, the Curie temperature $\left(\mathrm{T}_{\mathrm{C}}\right)$ must be higher than room temperature. The $T_{C}$ is also calculated using the mean-field approximation for the ferromagnetic cases by using formula ${ }^{76,77)}$ :

$$
\frac{3}{2} k_{B} T_{C}=-\frac{E \uparrow \uparrow-E \uparrow \downarrow}{N_{T M}}
$$

Where $\mathrm{N}_{\mathrm{TM}}=2$ is the number of TM impurities in the supercell ${ }^{78)}$. By using the formula, the Curie temperature is recorded higher that than the room temperature which propose the possibility of transition metal doped plumbene for spintronics application.

\subsection{Mechanical Properties}

As of late, molecular dynamics simulations under tensile loading, is used to assess plumbene's mechanical characteristics. The mechanical properties of plumbene sheets i.e. ultimate tensile strength and Young's modulus is calculated as $12-17 \mathrm{MPa}$ and $16 \mathrm{GPa}$ respectively which is predominant than bulk lead. These values of Young's modulus and ultimate tensile strength have been estimated under various temperatures and strain velocity formations and are found to be larger than bulk lead ${ }^{75,79)}$.

Henceforth, Plumbene with high mechanical stability may be used as reinforcing agent to improve various nanocomposite shaving high strength and exceptional material properties ${ }^{80-89)}$.

\section{Challenges and conclusion}

The absence of process in effortless synthesis techniques has restricted the exploratory developments to help the majority of the theoretical predictions. As of now, the existing synthesis approaches in fabricating Group IV elemental materials, similar to graphene, have been restricted to substrate reinforce epitaxial growth. Such constraint is anticipated because of the metastable nature of these elemental sheets. Other methods to provide such $2 \mathrm{D}$ sheets and cutting them off their growth substrates are however to be investigated. In that capacity, developments in synthesis techniques for generating 2D elemental sheets resembling graphene will be a huge achievement proceeding their enormous possibility for the advancement of another era of electronic, optoelectronic and spintronic applications. Undoubtedly, altogether cases, developing a synthesis method which is cost effective and finding a suitable semiconducting or insulating substrate for IV group 2D materials growth on a large scale stay challenging tasks for technological applications.

Thus, clearly, these $2 \mathrm{D}$ materials are guaranteed to a splendid future. However, the indication so far is encouraging. Up until now, plumbene seems to be like the next big thing in two-dimensional materials. Plumbene could be a possible candidate for processes related to high temperature applications and as a topological insulator. In comparison to graphene and silicene, plumbene has invaluable special properties due to its structural configurations too. Indeed, it has prevalent characteristics that can be useful for the functions related to machine traditional lead is utilized so far. Plumbene can too utilized for power generations, preparation of plumbenereinforced manufacturing, shipbuilding, and in batteries etc., wherein nanocomposites, etc. Plumbene will have anticipated uses wherein conductivity is the major issue.

\section{References}

1) K.S. Novoselov, A.K. Geim, S.V. Morozov, D.Jiang, S.V. Dubonos, I.V. Grigorieva, A.A. Firsov, "Electric field effect in atomically thin carbon films”,Science., 306(5696) 666-669 (2004). doi: 10.1126/science.1102896.

2) A. K. Geim, and K.S. Novoselov, "The rise of graphene”, Natural Materials., 6(3) 183191(2007).doi:10.1142/9789814287005_0002.

3) S.Y. Zhou, G.H. Gweon, A. V. Fedorov, P.N. First, W.A. de Heer, D. H. Lee, F. Guinea, A. H. Castro Neto, and A.Lanzara, "Substrate-induced band-gap opening in epitaxial graphene”, Nature Materials, 6770-775(2007).doi:10.1038/nmat2003.

4) K. Kim, J.Y.Choi, T. Kim, S.H.Cho, andH.J. Chung, "A role for graphene in silicon-based semiconductor devices”, Nature, 479(7373) 338-344 (2011). doi: 10.1038/nature10680.

5) S. Z. Butler, "Progress, challenges, and opportunities in two-dimensional materials beyond graphene". ACS Nano., 72898-2926 (2013). doi: 10.1021/nn400280c.

6) S. Cahangirov, M. Topsakal, E. Aktürk, H. Şahin, and D S. Ciraci, "Two- and One-Dimensional Honeycomb Structures of Silicon and 
Germanium", Physical Review Letters., 102236804 (2009). doi:10.1103/PhysRevLett.102.236804.

7) B. Aufray, A. Kara, S.B. Vizzini, H. Oughaddou, C. LéAndri, B. Ealet, and G.Le Lay, "Graphene-like silicon nanoribbons on $\operatorname{Ag}(110)$ : “A possible formationofsilicene", Applied Physics Letters., 96 (18)183102. (2010).doi:10.1063/1.34199 32.

8) S.Junki, Y. Tsuyoshi, N. Kan, and H.Hiroyuki "Epitaxial growth of silicene on ultra-thin Ag(111) films", New J. Phys., 16 (9) 095004. (2014). doi:10.1088/1367-2630/16/9/095004.

9) Isya Fitri Andhika, T. E. Saraswati and S. Hastuti, "The Structural Characteristics of Carbon Nanoparticles Produced by Arc Discharge in Toluene without Added Catalyst or Gases”, Evergreen Joint Journal of Novel Carbon Resource Sciences \& Green Asia Strategy, 7 (3) 417-428 (2020). doi: 10.5109/4068622

10) M.E. Dávila,"Germanene: a novel two-dimensional germanium allotrope akin to graphene and silicene", New J. $\quad$ Phys., 16 (9) 095002 (2014). doi:10.1088/1367-2630/16/9/095002.

11) M.E. Dávila, and G.L. Lay,"Few layer epitaxial germanene: A novel twodimensionalDiracmaterial", Science, Rep. 6 20714 (2016). doi:10.1038/srep20714.

12) H. Liu, T.N. Adam, Z. Zhen, X. Xu, and T. David, "Phosphorene: A new 2D material with high carrier mobility”, ACS Nanoscale Physics. 840334041(2014).doi.org/10.1021/nn505868h.

13) H. O. H. Churchill, P. Jarillo-Herrero, "Twodimensional crystals: phosphorus joins the family", Nature Nanotechnology, 9(5)330331(2014).doi:10.1038/nnano.2014.85.

14) E. S. Reich, "Phosphorene excites materials scientists”, Nature, 6 506(7486) 19 (2014). doi: 10.1038/506019a.

15) M. G. Chaudhary, N. Kumar, Shekhar, Sudheer, and R. Solanki, “A Ray of Hope for Future Technology: Phosphorene, Indian Journal of Science and Technology, 10(19) 1-17 (2017).doi: 10.17485/ijst/2017/v10i19/99528.

16) H. O. Churchill, and P. Jarillo-Herrero,"Twodimensional crystals: phosphorus joins the family”,NatureNanotechnology, 9(5) 330-331 (2014).doi: 10.1038/nnano.2014.85.

17) S. Saxena, R.P. Chaudhary, and S. Shukla, "Stanene: Atomically Thick Free-standing Layer of 2D Hexagonal Tin", Sci. Rep., 631073 (2016). doi:10.1038/srep31073.

18) C. Cesare, "Physicists announce graphene's latest cousin: stanene", Nature News, 524 (7563) 18 (2015). doi:10.1038/nature.2015.18113.

19) Z. Feng-feng, C. Wei-jiong,Y. Xu, G. Chun-lei, G. Dan-dan,L. Can-hua, D. Qian, Z. Shou-Cheng, and J. Jin-feng, "Epitaxial growth of two-dimensional stanene", Nature Materials, 14 (10)1020-1025. (2015). doi:10.1038/nmat4384.

20) J. Gao, G. Zhang, and Y.W. Zhang, "Exploring Ag (111) Substrate for Epitaxially Growing Monolayer Stanene: A First-Principles Study", Sci.Rep., 629107(2016). doi:10.1038/srep29107

21) J. Yuhara, Y. Fujii,K. Nishino, N. Isobe, M. Nakatake, L. Xian, A.Rubio, and G. L. Lay, "Large area planar staneneepitaxially grown onAg(111)", 2DMaterials, 5 (2) 025002 (2018). doi:10.1088/2053-1583/aa9ea0.

22) X.L. Yu, L. Huang, and J. Wu, "From a normal insulator to a topological insulator in plumbene”,Phys.Rev.B95125113(2017).doi.org/10.1 103/PhysRevB.95.125113

23) K. Kim, J.Y. Choi, T. Kim, S. H. Cho, and H.J.Chung, "Arole for graphene in silicon-based semiconductor devices”, Nature, 479(7373), 338-344 (2011). doi:10.1038/nature10680.

24) X. Ding, H. Liu, and Y. Fan, "Graphene-based materials in regenerative medicine”, Advanced healthcare materials, 4(10) (2015). doi:10.1002/adhm.201500203

25) F.M.P. Tonelli, V.A.M. Goulart, K.N. Gomes, and M.S. Ladeira, "Graphene-based nanomaterials: biological and medical applications and toxicity”, Nanomedicine (Lond), 10(15) 2423-2450 (2015). doi:10.2217/nnm.15.65.

26) S. Kumar, and K. Chatterjee, "Comprehensive review on the use of graphene-based substrates for regenerative medicine and biomedical devices”, ACS appl. Mater. Interfaces, 8(40) 26431-26457 (2016).doi:10.1021/acsami.6b09801.

27) S.Priyadarsini, S. Mohanty, S. Mukherjee, and S. Basu, "Graphene and graphene oxide as nanomaterials for medicine and biology application”, Journal of Nanostructure in Chemistry, 8 123-137 (2018). doi: 10.1007/s40097-018-0265-6.

28) K.I. Bolotin, K.J. Sikes, Z. Jiang, and M. Klima, G. Fudenberg,J.Hone,P.Kim, andH.L.Stormer, "Ultrahigh electron mobility in suspended graphene”,Solid State Communications, 146 9-10 351-355 (2008). doi:10.1016/j.ssc.2008.02.024.

29) B. Zhang, J. Zhang, X. Sang, C. Liu, T. Luo, L Peng, B. Han, X. Tan, X. Ma, D. Wang, and N. Zhao, "Cellular graphene aerogel combines ultralow weight and high mechanical strength: A highly efficient reactor for catalytic hydrogenation”, Sci. rep., 6 25830(2016). doi: 10.1038/srep25830.

30) D.S. Ghosh, I. Calizo, D. Teweldebrhan, E. P. Pokatilov, D. L. Nika, A. Balandin, W. Bao, F. Miao, and C. N. Lau, "Extremely high thermal conductivity of graphene: Prospects for thermal management applications in nanoelectronic circuits”, Appl. $\quad$ Phys. Lett., $92 \quad 151911$ (2008).doi:10.1063/1.2907977.

31) D.E. Sheehy, and J. Schmalian, “Optical transparency of graphene as determined by the fine-structure 
constant”, Phys. Rev. B80 193411 (2009). doi:10.1103/PhysRevB.102.125421.

32) U. Stöberl, U. Wurstbauer, W. Wegscheider, D. Weiss, and J. Eroms, "Morphology and flexibility of graphene and few-layer graphene on various substrates”,Applied Physics Letters, 93(5) 051906 (2008). doi:10.1063/1.2968310.

33) C. Cao, M. Daly, C.V. Singh, Y. Sun, and T. Filleter, "High strength measurement of monolayer graphene oxide Carbon”, Carbon, 81 497-504 (2015). doi:10.1016/j.carbon.2014.09.082.

34) M.F. Craciun, S. Russo, M. Yamamoto, and S. Tarucha, "Tuneable electronic properties in graphene”, Nano Today, 6(1) 42-60 (2011). doi:10.1016/j.physe.2011.10.007.

35) S. Konschuh, M. Gmitra, and J. Fabian, “Tightbinding theory of the spin-orbit coupling in graphene”, Phys. Rev.B, 82(24) (2010).doi: PhysRevB.82.245412.

36) M. Gmitra, D. Kochan, and J. Fabian, "Spin-orbit coupling in hydrogenated graphene”, Phys. Rev. Lett., 110(24) 246602 (2013).doi:PhysRevLett.110.246602.

37) A. Saffarzadeh, and R. Farghadan, "A spin-filter device based on armchair graphene nanoribbons", Applied Physics Letters, 98(2) (2011).doi:10.1063/1.3537965.

38) L. Kou, B. Yan, F. Hu, S.C. Wu, T.O. Wehling, C. Felser, C. Chen, and T. Frauenheim, "Graphenebased topological insulator with an intrinsic bulk band gap above room temperature”,NanoLett., 1312 6251-6255 (2013).doi:10.1021/nl4037214.

39) M.Z. Hasan, and C.L. Kane, "Colloquium: Topological insulators”, Rev. Mod. Phys., 823045 (2010). doi:10.1103/RevModPhys.82.3045.

40) Y. Ando, “Topological insulator materials”, J. Phys. $\begin{array}{llll}\text { Soc. } & \text { Jpn., } & 82 & 102001\end{array}$ (2013).doi:10.7566/JPSJ.82.063713.

41) S. Balendhran, S. Walia, H. Nili, S. Sriram, and M. Bhaskaran, "Elemental analogues of graphene: Silicene, germanene, stanene, and phosphorene”, Small, 11(6) 640-652 (2015).doi: 10.1002/smll.201402041.

42) X.L. Qi, and S.C. Zhang, "The quantum spin Hall effect and topological insulators”, Physics Today, 63 33-38 (2010).doi:10.1063/1.3293411.

43) H. Şahin, S. Cahangirov, M. Topsakal, E. Bekarogl, E.Aktrk, R.T. Senger, and S. Ciraci, "Monolayer honeycomb structures of group-IV elements and III$\mathrm{V}$ binary compounds: First-principles calculations”,Phys. Rev. B, 80(15) 155453 (2009).doi:10.1103/PhysRevB.80.155453.

44) M.I. Katsnelson, "Graphene: carbon in two dimensions”, Materials today, 10 (1-2) 20-27 (2007).doi:10.1016/S1369-7021 (06)71788-6.

45) D. Gray, A. McCaughan, and B. Mookerji, "Crystal structure of graphite, graphene and silicon”, Physics for Solid State Applications, 6730 (2009).
46) Y. Li, J. Zhang, B. Zhao, Y. Xue, and Z. Yang,"Constructive coupling effect of topological states and topological phase transitions in plumbene”,Phys. Rev. B., 99 195402(2019). doi:10.1103/PhysRevB.99.195402.

47) H. Zhao, C. W. Zhang, J. Wei-xiao, R. W. Zhang, S.S. Li, S. S. Yan, B. Zhang, P. Li, and P. Wang, "Unexpected Giant-Gap Quantum Spin Hall Insulator in ChemicallyDecoratedPlumbene Monolayer", Sci. Rep.6 20152 (2016). doi:10.1038/srep20152.

48) S. Mahmud and Md. K. Alam, "Large bandgap quantum spin Hall insulator inmethyl decorated plumbene monolayer: a first principles study”, RSC Advances, 9(72) 42194-42203 (2019).doi:10.1039/C9RA07531C.

49) L. Zhang, H. Zhao, J. Wei-xiao, C. W. Zhang, P. Li, and P.J. Wang "Discovery of a new quantum spin Hall phase in bilayer plumbene", Chem. Phys. Lett.,712(16) 78-82 (2018).doi:10.1016/j.cplett.2018.09.016.

50) Y. Xu, "Large-Gap Quantum Spin Hall Insulators in Tin Film”, Phys. Rev. Lett. 111136804 (2013). doi:10.1103/PhysRevLett.111.136804.

51) H. Zhang, Z. Wang and $\mathrm{X}$. Xu, "Room temperature quantum spin Hall insulator: Functionalized stanene on layered PbI2 substrate”, Appl. Phys. Lett., 111 072105 (2017). doi:10.1063/1.4985643.

52) M. Zhou, W. Ming, L. Zheng, Z. Wang, Y. Yao , F. Liu, "Formation of quantum spin Hall state on $\mathrm{Si}$ surface and energy gap scaling with strength ofspinorbitcoupling“, Sci Rep., 4 71(2014).doi: 10.1038/srep07102.

53) C. Si, J. Liu, Y. Xu, J. Wu, B. L. Gu, and W. Duan, "Functionalized germanene as a prototype of largegap two-dimensional topological insulators”, Phys. Rev. B. 89, $115429 \quad$ (2014). doi:10.1103/PhysRevB.89.115429.

54) R.W.Zhang, C.W. Zhang, W.X.Ji, P.Li, P.J.Wang, S.S.Li, and S.S.Yan, "Silicon-based chalcogenide: Unexpected quantum spin Hall insulator with sizable band gap”, Appl. Phys. Lett. 109, 182109 (2016). doi:10.1063/1.4966124.

55) X.L. Yu and J. Wu, "Evolution of the topological properties of two-dimensional group IVA materials and device design", Physical Chemistry Chemical Physics, 20(4) (2018). doi:10.1039/C7CP07420D.

56) Q. Zhong, L. Kong, J. Gou, W. Li, S. Sheng, S. Yang, P. Cheng, H. Li, K. Wu, and L. Chen, "Synthesis of BoropheneNanoribbons on Ag(110) Surface”, Phys. Rev. Materials 1, 021001(2017). doi:10.1103/PhysRevMaterials.1.021001.

57) A.J. Mannix, Z. Zhang, N.P. Guisinger, B.I. Yakobson, and M.C.Hersam, "Borophene as a prototype for synthetic 2D materials development”, Nature Nanotechnology, 13 444-450(2018). doi:10.1038/s41565-018-0157-4. 
58) B. Kiraly, X. Liu, L. Wang, Z. Zhang, A.J. Mannix, L. F. Brandon, B.I. Yakonson, M.C.Hersam, and N.P. Guisinger, "Borophene synthesis on Au (111)", ACS Nano, 134 3816-3822(2019). doi:10.1021/acsnano.8b09339.

59) A.H. Woomer, T.W. Farnsworth, J. Hu, R.A. Wells, C.L.Donley, and S.C.Warren, "Phosphorene: synthesis, scale-up, and quantitative optical spectroscopy”, ACS Nano, 9988698884(2015).doi:10.1021/acsnano.5b02599.

60) M. Fortin-Deschênes, O. Waller, T.O. Mentes, A. Locatelli, S. Mukherjee, F. Genuzio, P.L.Levesque, A. Hebert, R. Martel and O. Moutanabbir, "Synthesis of antimonene on germanium” Nano Lett., 178 4970-4975 doi:10.1021/acs.nanolett.7b02111.

(2017).

61) L. Lu, Z. Liang, L. Wu, Y.X. Chen, Y. Song, S. C. Dhanabalan, J. S. Ponraj, B. Dong, Y. Xiang, F. Xing, D. Fan, H. Zhang, "Few-layer bismuthene: sonochemical exfoliation, nonlinear optics and applications for ultrafast photonics with enhanced stability”, Laser \& PhotonicsRev.,12(1)(2018).doi:10.1002/lpor.201700 221.

62) J. Yuhara, and G. L. Lay, "Beyond silicene: synthesis of germanene, stanene and plumbene”, Jpn. J. Appl. Phys. 59 SN0801 (2020).doi:10.35848/13474065/ab8410.

63) W. N. Septiadi, K. Astawa, I.G. A. P. Arvikadewi, D. Febraldo, and G. J. P. Putra, "Boiling Phenomenon of Graphene Nano-Coating Wick Heat Pipe”, Evergreen Joint Journal of Novel Carbon Resource Sciences \& Green Asia Strategy, 7(2) 297-302 (2020). doi: 10.5109/4055236

64) Z. Qiao, W.K. Tse, H. Jiang, Y. Yao, Q. Niu, “Twodimensional topological insulator state and topological phase transition in bilayer graphene”, Phys. Rev. Lett., 107, 256801(2011).doi:10.1103/PhysRevLett.107.256801

65) M. Ezawa, “A topological insulator and helical zero mode in silicene under an inhomogeneous electric field”, New J. Phys. 14033003 (2012).doi: 10.1088/1367-2630/14/3/033003.

66) M. S. Rahman, T. Nakagawa, and S. Mizuno, " Germanene: Experimental Study for Graphene like Two Dimensional Germanium”, Evergreen Joint Journal of Novel Carbon Resource Sciences \& Green Asia Strategy, 1(2) 25-29 (2014).doi: 10.5109/1495160.

67) C. Huang, J. Zhou, H. Wu, K. Deng, P. Jena and E. Kan, "Quantum phase transition in germanene and stanene bilayer: from normal metal to topological insulator”, J. Phys. Chem. Lett.,71019191924(2016).doi:10.1021/acs.jpclett.6b00651.

68) H. K.Kaushik, S. Kumar, M.G. Chaudhary, S. Khan, "Optical properties of CdS: Pb thin layer deposited on glass substrate”, Indian Journal of Pure and Applied Physics, 58(01) 11-15 (2020).

69) M. Ezawa "Monolayer Topological Insulators: Silicene, Germanene, and Stanene”, J. Phys. Soc. Jpn., 84,121003(2015).doi:10.7566/JPSJ.84.121003.

70) Z. X. Pang, W. Yong, W. X. Ji, C.W. Zhang, P.J. Wang, and P. Li, "Two-dimensional ligandfunctionalized plumbene: A promising candidate for ferroelectric and topological order with a large bulk band gap”,Physica E: Low-dimensional Systems and Nanostructures,120,114095(2020).doi:10.1016/j.phy se.2020.114095.

71) B. Gustav, S. Jonas, K. André, B. Stefan, K. V. Bergmann, and R. Wiesendanger,"Plumbene on a Magnetic Substrate: A Combined Scanning Tunneling Microscopy and Density Functional Theory Study”,Phys. Rev. Lett., 124126401 (2020). doi:10.1103/PhysRevLett.124.126401.

72) Y.P. Feng, L. Shen, M. Yang, A. Wang, M. Zeng, Q. Wu, S. Chintalapati, and C. R. Chang, "Prospects of spintronics based on 2D materials", Materials Science, Wiley Interdisciplinary Reviews: Computational Molecular Science, 7(5) e1313(2017). doi:10.1002/wcms.1313.

73) R. Bratschitsch, I. B. Villamil, and R Schmidt, "Inverted valley polarization in optically excited transition metal dichalcogenides”, Nature Communications, 9(971) (2018). doi:10.1038/s41467-018-03354-1.

74) D. Hashemi, and H. Iizuka, "Magnetic properties of 3d transition metal (Sc-Ni) doped plumbene”, RSC Advances, 10(12) 6884-6892 (2020). doi: 10.1039/C9RA10337F.

75) J. Kudrnovsky”, I. Turek, V. Drchal, F. Maca, P. Weinberger, and P. Bruno, "Exchange interactions in III-V and group-IV diluted magnetic semiconductors”, Physical review, B69 115208 (2004). doi: 10.1103/PhysRevB.69.115208.

76) F. Maca, J. Kudrnovsky', V. Drchal, and G. Bouzerar, "Magnetism without magnetic impurities in $\mathrm{ZrO}_{2}$ oxide”, Applied Physics Letters, 92212503 (2008). doi: 10.1063/1.2936858.

77) Y. C. Cheng,1 Z. Y. Zhu,1 W. B. Mi,2 Z. B. Guo,3 and U. Schwingenschl"ogl1,*, "Prediction of twodimensional diluted magnetic semiconductors: Doped monolayer $\mathrm{MoS}_{2}$ systems”, Physical review, B87 100401 (2013). doi: 10.1103/PhysRevB.87.100401.

78) D. K. Das, J. Sarkar, S. K. Singh, "Effect of sample size, temperature and strain velocity on mechanical properties of plumbene by tensile loading along longitudinal direction: A molecular dynamics study”, Computational Materials Sciences, 151 196-203 (2018). doi: 10.1016/j.commatsci.2018.05.006.

79) D.K.Das, and S. K. Singh, "Plumbene: A New 2DMaterial Resembling Graphene”, Advances in 
Industrial and Production Engineering, 193-197 (2018).doi:10.1007/978-981-13-6412-9_18

80) K. Chihara, M. K. Ito, Kitajau, and S. Okada, " Cathode Property of $\mathrm{Na} \_2 \mathrm{C} \_\mathrm{xO} \_\mathrm{x}[\mathrm{x}=4,5$, and 6] and K_2C_6O_6 for Sodium-ion Batteries", Evergreen Joint Journal of Novel Carbon Resource Sciences \& Green Asia Strategy, 4(1) 1-5 (2017). doi:10.5109/1808304.

81) S. K. Kim and R. Hattori, "Study of Contact Resistance on Organic Thin Film Transistor with Surface Treatments", Evergreen Joint Journal of Novel Carbon Resource Sciences \& Green Asia Strategy, 2(1) 1-5 (2015). doi:10.5109/1500421.

82) K. Tewari and R. Dev, " Analysis of Modified Solar water Heating System Made of Transparent Tubes \& Insulated Metal Absorber", Evergreen Joint Journal of Novel Carbon Resource Sciences \& Green Asia Strategy, 5(1) 62-72 (2018). doi:10.5109/1929731.

83) M. Sultan, I. I. El-Sharkawl, T. Miyazaki, B. B. Saha, and S. Koyama, "Experimental Study on Carbon Based Adsorbents for Greenhouse Dehumidification ", Evergreen Joint Journal of Novel Carbon Resource Sciences \& Green Asia Strategy, 1(21) 5-11 (2014). doi:10.5109/1495157.

84) R. A. Rouf, M. A. H. Khan, K. M. A. Kabir, and B. B. Saha, "Energy Management and Heat Storage for Solar Adsorption Cooling”, Evergreen Joint Journal of Novel Carbon Resource Sciences \& Green Asia Strategy, 3 (2) 1-10 (2016). doi: 10.5109/1800866.

85) M. R. Zakaria, M. F. Omar, H. Md. Akil, and M. M. Al. B. Abdullah, "Study of Carbon Nanotubes Stability in Different Types of Solvents for Electrospray Deposition Method”, Evergreen Joint Journal of Novel Carbon Resource Sciences \& Green Asia Strategy, $7 \quad$ (4) 538-543 (2020). doi: 10.5109/4150473.

86) A. F. Ridassepri, F. Rahmawati, K.R. Heliani, Chairunnisa, J. Miyawaki, and A. T. Wijayanta, "Activated Carbon from Bagasse and its Application for Water Vapor Adsorption”, Evergreen Joint Journal of Novel Carbon Resource Sciences \& Green Asia Strategy, 7(3) 409-416 (2020). doi: 10.5109/4068621.

87) A. Gupta, H. Kumar, L. Nagdeve and P. K. Arora, "EDM Para metrics Study of Composites Materials: A review”, Evergreen Joint Journal of Novel Carbon Resource Sciences \& Green Asia Strategy, 7(4) 519529 (2020). doi: 10.5109/4150471.

88) J.A. Hidayat and S. Bambang, "Characteristics, Structure and Morphology of Carbon Deposit from Biodiesel Blend", Evergreen Joint Journal of Novel Carbon Resource Sciences \& Green Asia Strategy, 7(4) 609-614 (2020). doi: 10.5109/4150514.

89) M. Alimin, A. Syukri, A. Hairul and Emriadi, "Mechanical Properties and Biodegradibility of Areca Nut Fiber-Reinforced Polymer Blend Composites", Evergreen Joint Journal of Novel
Carbon Resource Sciences \& Green Asia Strategy, 7(3) 366-372 (2020). doi: 10.5109/4068618. 